1 An intercomparison exercise of good laboratory practices for nano2 aerosols size measurements by mobility spectrometers

4 F. Gaie-Levrel ${ }^{*}$, S. Bau ${ }^{2}$, L. Bregonzio-Rozier ${ }^{1}$, R. Payet $^{2}$, S. Artous ${ }^{3}$, S.

5 Jacquinot $^{3}$, A. Guiot ${ }^{3}$, F.-X. Ouf ${ }^{4}$, S. Bourrous ${ }^{4}$, A. Marpillat ${ }^{5}$,

6 C. Foulquier ${ }^{5}$, G. Smith ${ }^{5}$, V. Crenn ${ }^{5}$, N. Feltin ${ }^{1}$

$7{ }^{1}$ LNE - 1 rue Gaston Boissier, 75724 Paris Cedex 15, France

$8{ }^{2}$ INRS - 1, rue du Morvan - CS 60027, 54519 Vandoeuvre Cedex, France

$9{ }^{3}$ Univ. Grenoble Alpes - CEA Grenoble - PNS, 17 rue des martyrs 38054 Grenoble

10 Cedex, France

$11{ }^{4}$ Institut de Radioprotection et de Sûreté Nucléaire (IRSN), PSN-RES, SCA, Gif-sur-

12 Yvette, 91192, France

$13{ }^{5}$ ADDAIR - 189 rue Audemars, 78530 Buc, France

$14 *$ francois.gaie-levrel@lne.fr 


\section{An intercomparison exercise of good laboratory practices for nano- 2 aerosols size measurements by mobility spectrometers}

An intercomparison campaign on nanoparticle size measurement was organized in the frame of the French nanoMetrology club. The aim of this study is to make an inventory of the metrological capabilities of all measurement techniques in France involved in the "nano" size range, including the SMPS (Scanning Mobility Particle Sizer) concerning aerosol metrology. For this study, four samples have been proposed, namely (1) - a $\mathrm{SiO}_{2}$ colloidal suspension (FD304) consisting of a monomodal population, (2) two samples consisting of two nanoparticle populations of $\mathrm{SiO}_{2}$ having proportions to be determined and (3) - a $\mathrm{TiO}_{2}$ colloidal suspension. Ten SMPS associated to five participants around a common experimental setup were performed in link with a control SMPS to have simultaneous measurements with a same instrument in each laboratory in parallel with the SMPS used by each partner. This article presents SMPS results of this study associated with the description of the experimental set-up and the sample preparation protocol with an identified schedule and comparison with SEM measurements. The present paper does not focus on the actual capability of the tested mobility spectrometers, but aims to highlights the good laboratory practices using their own but common resources in terms of aerosol generation and measurement set-ups.

Keywords: Intercomparison, nano-aerosols, colloidal suspension, SMPS

\section{Introduction}

Atmospheric aerosols are known to have a large impact on human health (Vedal et al., 2006; Lawrence et al., 2007), atmospheric chemistry and climate (Forster et al., 2007). Concerning human exposure in workplace and outdoor environments, aerosol inhalation can cause adverse health effects due to the deposition of airborne particles in the respiratory tract (ICRP, 1994). In addition to particle chemistry/chemical composition, such impacts are mainly dependent on particle number size distribution (PNSD), which can be measured by instrumental techniques such as Mobility Particle Size Spectrometer (MPSS), also known as Differential Mobility Particle Sizer (DMPS), Differential Electrical Mobility Analyzer (DEMA) or Scanning Mobility Particle Sizer (SMPS). Such instruments are composed of a Differential Mobility Analyzer (DMA) with a bipolar diffusion charger (aerosol neutralizer), used to select a given particle size, coupled to a Condensation Particle Counter (CPC) allowing particles to be counted individually. Upstream the DMA, an impactor is used to remove larger particles which could disturb neutralizing process and lead to biases in size distribution measurements. Although not best suited for field measurement campaigns (Leskinen et al., 2012; Bau et al., 2013), SMPS is a standardised method already used in the certification process of spherical particle reference materials.

Several interlaboratory comparisons of SMPS have been performed over the last 25 years, mainly in the context of atmospheric measurements (Wiedensohler et al. 1993 ; Khlystov et al. 2001 ; Rodrigue et al. 2007 ; Helsper et al. 2008; Jeong and Evans, 2009 ; Watson et al., 2011; Wiedensohler et al., 2012; Motzkus et al. 2013; Gómez-Moreno et al. 2015; Fonseca et al., 2016; Wiedensohler et al., 2017). Joshi et al. (2012) conducted a metrological study of two different SMPS on both ambient and laboratory test aerosols. Even though they reported an excellent consistency while comparing the mean size and geometric standard deviation from both instruments, the authors highlight the necessity to perform intercomparison exercises for harmonisation of the measurements.

Laboratory performances of four DMAs were evaluated by Fissan et al. (1996) by sampling monodisperse aerosols in the 6-50 $\mathrm{nm}$ size range under the same operating conditions for each system, such as sample and sheath air flow rates, input and tubing lengths. Their results provide a quantitative comparison of the mobility selection accuracy and diffusion losses of nanometer aerosols in such systems. Later, Dahmann et al. (2001) reported acceptable comparability of the results obtained in the framework of an international inter-comparison performed in Germany (11 models investigated). This study highlighted the need to guarantee uniform instrument parameters under conditions of good practice and user skills, in line with the conclusions drawn by Kaminski et al. (2013) from a laboratory study which showed a higher comparability between 8 SMPS models. In the paper from Asbach et al. (2009), the response of four different mobility particle sizers was investigated when measuring $\mathrm{NaCl}$ and diesel soot particles. The major conclusions of these inter-laboratory comparison studies are the lack of (1) measurement standards with specific recommendations and (2) harmonized and standardized measurement procedures. 
A lack of metrological traceability can be identified in these studies highlighting the crucial importance to have traceable parameters. Indeed, in the paper from Wiedensohler et al. (2012), the issue of the harmonization of measurement procedures to facilitate high quality long-term observations of atmospheric particle size number distributions obtained by SMPS is raised. In more recent work, Wiedensohler et al. (2017) state that an SMPS calibration facility should have one or several calibrated reference SMPS. For a complete quality assurance, it is therefore proposed that measurement procedures take into account (1) - sizing calibration of the candidate SMPS using a certified particle size standard, (2) - PNSD intercomparison of candidate SMPS against a reference SMPS, (3) - intercomparison of the integral particle number concentration (PNC) of the candidate SMPS against a calibrated reference CPC with pre-calibration of the candidate CPC counting efficiency curve. It is worth mentioning that PNSD measurement is also based on the commonly used equations for the bipolar charge equilibrium (Fuchs, 1963) as described in ISO 15900, which is agreed by convention (Wiedensohler et al., 2017) but not SItraceable

In industrial environments where workers are confronted to engineered nanomaterials, inhalation exposure to nano-objects aggregates/agglomerates (NOAAs) must be monitored with adapted techniques. In this context, other intercomparis on studies on PNSD measurements by SMPS were performed on $\mathrm{TiO}_{2}$ (Leskinen et al., 2012; Ding et al., 2016). Babick et al., (2016a,b) showed for several nano-additived materials that the SMPS number-weighted median diameters deviate from electron microscopy results by a factor not larger than 1.5 .

In this work, we present an SMPS intercomparison study, which is part of a multi-technical interlaboratory comparison of the measurement of the size of nano-objects performed in the framework of the French nanoMetrology Club (nMC). This nMC (Club nanoMétrologie, 2019) is coordinated since 2011 by LNE and represents a French network gathering around 400 members and dealing with the topic of material characterization at the nanoscale by creating bridges between academic and industry communities.

The overall objective of this large intercomparison is to make an inventory of the metrological measurement capabilities of various measurement techniques in France. This paper focuses on the results of 10 SMPS associated to five participants around a common experimental setup in link with a control SMPS. Four different samples were investigated: (1) - a $\mathrm{SiO}_{2}$ monomodal colloidal suspension of reference nanoparticles (ERM-FD304); (2) - two samples $\left(\mathrm{SiO}_{2}-1\right.$ and $\left.\mathrm{SiO}_{2}-2\right)$ consisting of two populations of $\mathrm{SiO}_{2}$ nanoparticles; (3) - a fourth sample consisting of $\mathrm{TiO}_{2} \mathrm{NOAAs}$ also called E171 as a food additive. The objective of this paper is not to characterize the actual capability of the tested mobility spectrometers, but to demonstrate the good laboratory practices using their own - but common - resources in terms of aerosol generation and measurement set-ups.

\section{Experimental setup and SMPS types}

A SMPS intercomparison exercise is highly constrained by the difficulty to move instruments at the same location in the same time especially when they use radioactive sources. The strategy chosen in this study was based on the reproduction of an experimental setup and measurement sequence, associated with a common measurement device. More precisely, each partner from Lab $n^{\circ} 1$ to Lab ${ }^{\circ} 4$ used its own resources to build the experimental set-up presented in Fig1. As a common measurement device, a PALAS control SMPS (Lab ${ }^{\circ} 5$ ) was used during this interlaboratory comparison in order to have simultaneous measurements performed with a same instrument in each laboratory in parallel with the SMPS used by each partner. The common experimental setup implemented by each partner for this study was based on aerosol generation thanks to an atomizer (model 3076, TSI) operated in recirculation mode, coupled to a diffusion dryer system (model 3062-NC, TSI), and a downstream dilution air flow delivering a constant flow rate of $8 \mathrm{~L} / \mathrm{min}$ allowing multiple measurements to be carried out in parallel (Fig 1). It is important to note that atomizer system was chosen for this study as the only generator owned by each participant by keeping in mind that the main objective of this study is to demonstrate the good laboratory practices using common resources in terms of aerosol generation and measurement set-ups and common sample preparation protocols. The particle-free air introduced in the setup was preliminarily dried and filtered by means of a specific device (model 3074B, TSI). 


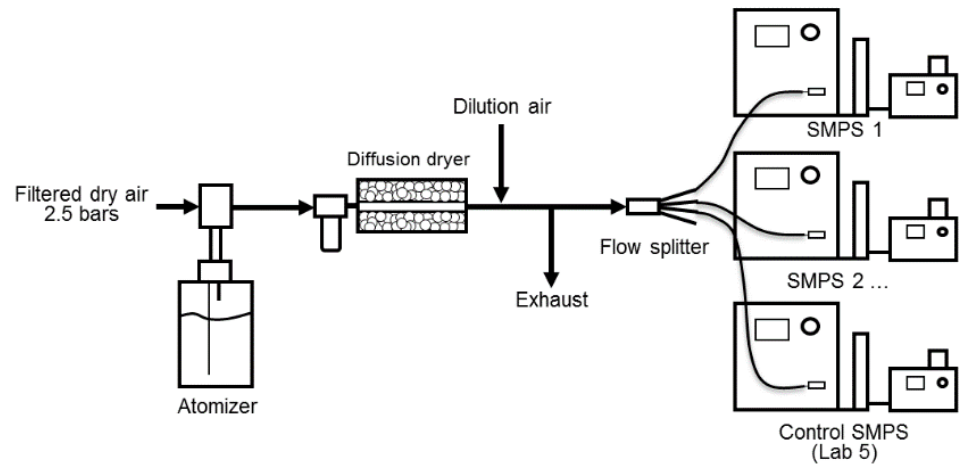

Fig 1: Experimental setup used by each partner for this study.

A four-way flow splitter (model 3708, TSI) was used to simultaneously provide the homogeneous aerosol distribution to the different SMPS operating in parallel, each of them was connected with the same length $(\sim 1 \mathrm{~m})$ of antistatic tubing. Particle losses within the sampling tubes were supposed to be equivalent due to the range of aerosol flow rates of the instruments; therefore, no correction for particle concentration was performed. Table 1 presents the technical specifications of the different SMPS involved in this study for each laboratory.

10 Table 1 shows that long columns were mainly used for particle classification. It is important to mention 11 that raw SMPS data are converted into PNSD by assuming that airborne particles reach the target charge 12 distribution imposed by the bipolar charger (Fuchs, 1963; Stolzenburg \& McMurry, 2008). This is 13 typically achieved using neutralization sources, which bring aerosols to a steady-state charge distribution, 14 whatever their initial state-of-charge.

Table 1: SMPS types implicated in this intercomparison study.

\begin{tabular}{|c|c|c|c|c|c|c|c|c|}
\hline & \multicolumn{2}{|c|}{ Company } & $\begin{array}{c}\text { DMA + } \\
\text { Column }\end{array}$ & Source & $\begin{array}{c}\text { CPC } \\
\text { working fluid / } \\
d_{50}(\mathrm{~nm})\end{array}$ & $\begin{array}{l}\text { Max con centration } \\
\text { for single particle } \\
\text { counting }\left(\# / \mathrm{cm}^{3}\right)\end{array}$ & $\begin{array}{c}\text { Sheath flow / } \\
\text { Sample flow } \\
\text { (L/min) }\end{array}$ & $\begin{array}{l}\text { Channels } \\
\text { / decade }\end{array}$ \\
\hline \multirow{3}{*}{ 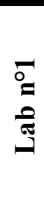 } & SMPS 1.1 & & $3080+$ long & ${ }^{85} \mathrm{Kr} 3077 \mathrm{~A}$ & $\begin{array}{c}3775 \\
\text { butanol / } 4\end{array}$ & $5 \times 10^{4}$ & \multirow{3}{*}{$3 / 0.3$} & \multirow{3}{*}{64} \\
\hline & SMPS 1.2 & T SI & $3082+$ nano & RX 3088 & $\begin{array}{c}3788 \\
\text { water / } 2.5\end{array}$ & $4 \times 10^{5}$ & & \\
\hline & SMPS 1.3 & & $3080+$ long & ${ }^{85} \mathrm{Kr} 3077$ & $\begin{array}{l}3775 \\
\text { butanol / } 4\end{array}$ & $5 \times 10^{4}$ & & \\
\hline \multirow{3}{*}{ 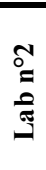 } & SMPS 2.1 & T SI & $3080+$ long & ${ }^{85} \mathrm{Kr} 3077$ & $\begin{array}{c}3785 \\
\text { water / } 4.2\end{array}$ & $<2 \times 10^{4}$ & $3 / 0.3$ & 64 \\
\hline & SMPS 2.2 & GRIMM & $\begin{array}{l}\text { Middle } \\
\text { Vienna }\end{array}$ & ${ }^{241} \mathrm{Am}$ & \multirow{2}{*}{$\begin{array}{c}5416 \\
\text { butanol / } 4\end{array}$} & \multirow{2}{*}{$1.5 \times 10^{5}$} & \multirow{2}{*}{$3 / 0.3$} & 36 \\
\hline & & & Long Vienna & RX XRC-05 & & & & 20 \\
\hline 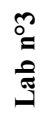 & SMPS 3.1 & T SI & $3082+$ long & RX 3088 & $\begin{array}{c}3787 \\
\text { water / } 5\end{array}$ & $2.5 \times 10^{5}$ & $6 / 0.6$ & 64 \\
\hline \multirow{3}{*}{ 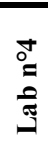 } & SMPS 4.1 & & $3080+$ long & ${ }^{85} \mathrm{Kr} 3077$ & \multirow{3}{*}{$\begin{array}{c}3775 \\
\text { butanol / } 4\end{array}$} & \multirow{3}{*}{$5 \times 10^{4}$} & $\begin{array}{l}15 / 1.5 \\
3 / 0.3\end{array}$ & \multirow{3}{*}{64} \\
\hline & SMPS 4.2 & T SI & $3080+$ long & RX 3087 & & & $3 / 0.3$ & \\
\hline & SMPS 4.3 & & $3080+$ long & RX 3088 & & & $15 / 1.5$ & \\
\hline 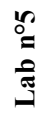 & $\begin{array}{l}\text { Control } \\
\text { SMPS }\end{array}$ & PALAS & $\begin{array}{c}\text { control unit } \\
\mathrm{DEMC}+\text { long }\end{array}$ & $\begin{array}{l}\text { RX XRC- } \\
049\end{array}$ & $\begin{array}{c}\text { Envi-CPC } 100 \\
\text { butanol / } 7\end{array}$ & $1 \times 10^{5}$ & $\begin{array}{c}9 / 0.9 \\
3.6 / 0.9 *\end{array}$ & 64 \\
\hline
\end{tabular}

18 In this study, radioactive $\left({ }^{85} \mathrm{Kr}\right)$ and soft X-ray sources were used to generate the bipolar ions that diffuse 19 onto the particles and bring them to the charge equilibrium. The bipolar charge equilibrium of Tigges et 20 al. (2015) was used for the soft X-ray charger in the SMPS inversion routine, while the one described by 21 Wiedensohler $(1986,1988)$ was used for the radioactive neutralizer as an approximation of the Fuchs 22 (1963) diffusion theory for particle sizes in the submicrometer range. It is important to mention that this 
study does not take into account neutralization efficiency difference between spherical nanoparticles and NOAAs as described by Lall \& Friedlander (2005).

Wiedensohler et al. (2017) showed that PNSD comparisons between a SMPS with a soft X-ray bipolar charger and a reference SMPS with a ${ }^{85} \mathrm{Kr}$ bipolar diffusion charger is excellent, i.e. in the $\pm 10 \%$ target uncertainty. Other work showed a good correlation in the particle number concentration and differences in the count median diameter and geometric standard deviation below $4 \%$ for a polydisperse $\mathrm{NaCl}$ aerosol measured with GRIMM SMPS equipped with either an X-ray source (TSI RX 3087) or a radioactive source (Nicosia et al., 2014). The results from Nicosia et al. (2018) confirmed that instrumental uncertainties introduced by the use of the X-ray sources rather than a radioactive neutralizer in the size range 10-300 $\mathrm{nm}$ are negligible, in line with previous work published by Kallinger et al. (2012).

Concerning CPC, water and butanol-based instruments were involved (w\&b-CPC respectively). Franklin et al. (2010) compared w\&b-CPC for diesel combustion aerosols. They pointed out a disagreement for one of the w-CPC, which was attributed to the use of water as a condensing fluid. This was also pointed out by the work of Bau et al. (2019) which highlighted that the accuracy of w-CPC is dependent on their technical design. To minimize instrumental or model differences, post-data correction equations were proposed by Lee et al. (2013) to adjust the data from TSI water-based CPC using exponential models. Bau et al. $(2017,2019)$ show that w\&b-CPC studied were found to be within $\pm 25 \%$ of the reference, except for a w-CPC (TSI model 3787) known to be highly sensitive to particle hydrophobicity. It is important to note that such parameter will have a minor effect in our study due to the sample nature.

As stated in Table 1, the main SMPS versions used in the aerosol measurement community are present in this intercomparison study, knowing that TSI models are mainly represented. For each SMPS involved, a sheath flow / sample flow (SSF) ratio of 10 was respected to ensure size resolution with an optimized DMA transfer function. Only the Lab $n^{\circ} 5$ control SMPS was used with a SSF ratio of 4 in the case of the $\mathrm{TiO}_{2}$ sample, in order to be capable of covering the range of particle diameter of interest. Indeed, a range of electrical mobility diameters from a few nanometers up to $200 \mathrm{~nm}$ was used for ERM-FD304, $\mathrm{SiO}_{2}-1$ and $\mathrm{SiO}_{2}-2$ samples; this range was increased up to $700 \mathrm{~nm}$ for the $\mathrm{TiO}_{2}$ sample.

\section{Samples, preparation protocol and data acquisition}

Concerning samples, ERM-FD304 was used as a Certified Reference Material (CRM) to verify instrument calibration and to adjust measurements when required. This CRM is characterized by a reference number-based modal diameter of $27.8 \pm 1.5 \mathrm{~nm}$ obtained by electron microscopy (EM), knowing that EM measurements correspond to the geometrical diameter of particles, which is not identical but equivalent to the electrical mobility diameter in this case of spherical particles (Kulkarni et al., 2011). $\mathrm{SiO}_{2}-1$ and $\mathrm{SiO}_{2}-2$ samples were specifically synthetized for this study as bi-populated colloidal suspensions. In parallel of particle size measurements, the determination of the proportions between the two populations was also asked to each participant to this study since both samples are characterized by two different populations proportions. The $\mathrm{TiO}_{2}$ sample used for this study was a foodgrade reference, also known as E171.

All stock samples, preliminary prepared by LNE for all techniques, were provided to all participants and stored at room temperature and protected from light. For each participant, preparation protocols for samples to be analyzed were deliberately basic (dilutions in ultrapure water, MilliQ, Millipore, 18.2 M $\Omega . c m$ resistivity) to be performed as simply as possible. Dilution factors of 750, 600, 500 and 111 for ERM-FD304, $\mathrm{SiO}_{2}-1, \mathrm{SiO}_{2}-2$ and $\mathrm{TiO}_{2}$ stock samples respectively were used. Atomizer cleaning and diffusion drier regeneration protocols were also provided to each participant.

As regards to data acquisition, each partner was required to record 5 scans for each sample, with a global duration of $3 \mathrm{~min}$ each and a waiting time of $2 \mathrm{~min}$ between each scan integrating a $20 \mathrm{~s}$ voltage decrease time. A blank level was checked between each scan using ultrapure water nebulization. For TSI SMPS, the AIM software was used by each partner while Grimm nanosoftware and PDAnalyze software were used for GRIMM and PALAS SMPS, respectively.

\section{Results}

\subsection{Particle number size distributions}

Figs 2 to 5 show the average PNSD measured by the different SMPS for aerosols generated by atomization of samples ERM-FD304, $\mathrm{SiO}_{2}-1, \mathrm{SiO}_{2}-2$ and $\mathrm{TiO}_{2}$, respectively. The error bars correspond to the standard deviations calculated on the five measurements made for each sample $(k=1)$. The PNSD were normalized with regards to the total number concentration of particles above $20 \mathrm{~nm}$ measured by each SMPS for each sample to take into account the different size range of the SMPS involved. For all 
1 samples, optimization of sample preparation and dilution protocol was performed during a pilot 2 laboratory study prior the inter-laboratory exercise. In spite of multiple tests, it remained unsuccessful to avoid multiplets generation around 40nm using the atomizer generator for the ERM-FD304 sample (Fig2). For the same sample, the larger error bars associated with the PNSD provided by SMPS5.1@Lab4 are due to a technical problem during the test session. Note that in the case of bimodal suspensions $\left(\mathrm{SiO}_{2}\right.$ $1, \mathrm{SiO}_{2}-2$ samples), the contribution as sociated with the MilliQ water nebulization induces the presence of a non-negligible background aerosol and limits the identification of the first population of these samples. PNSD of MilliQ water only are available in the appendix associated to this paper (Fig8). Therefore, it was not possible to calculate the ratios between the two populations of these bi-modal samples. For $\mathrm{SiO}_{2}-2$ sample (Fig4), the error bars associated with empty symbols for SMPS 4.1 size distribution $(>180 \mathrm{~nm})$ were deleted due to their large contributions.
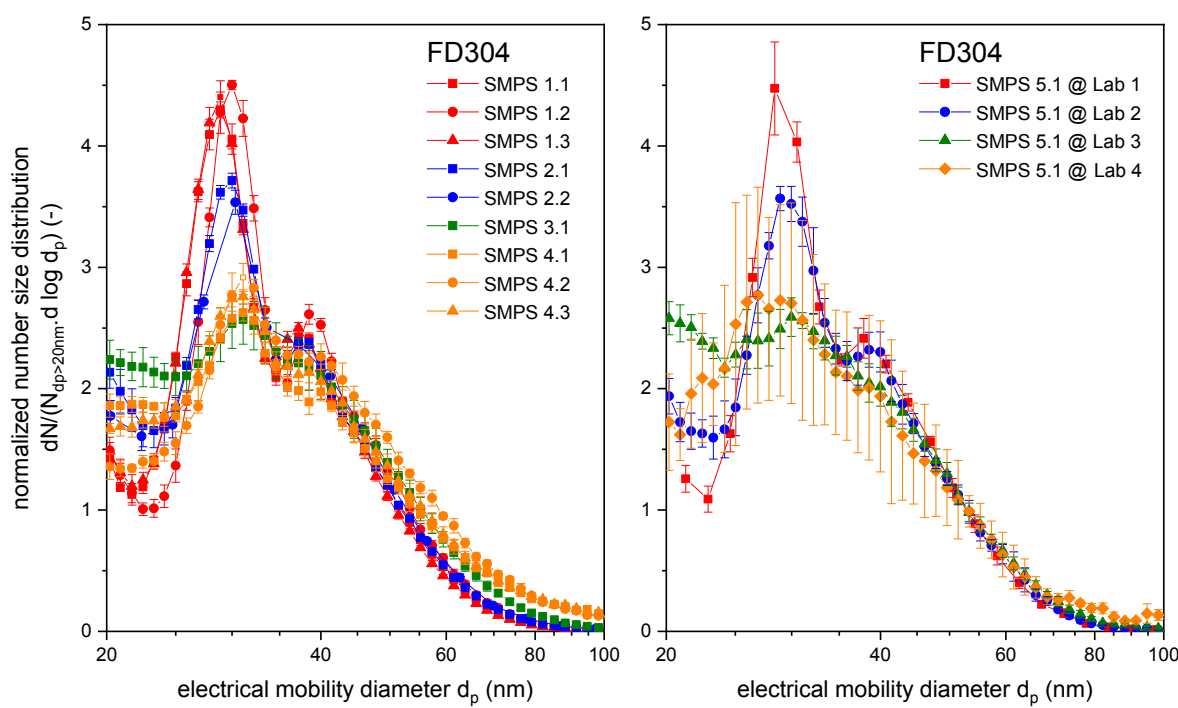

Fig2: Average particle number size distributions for the ERM-FD304 sample.
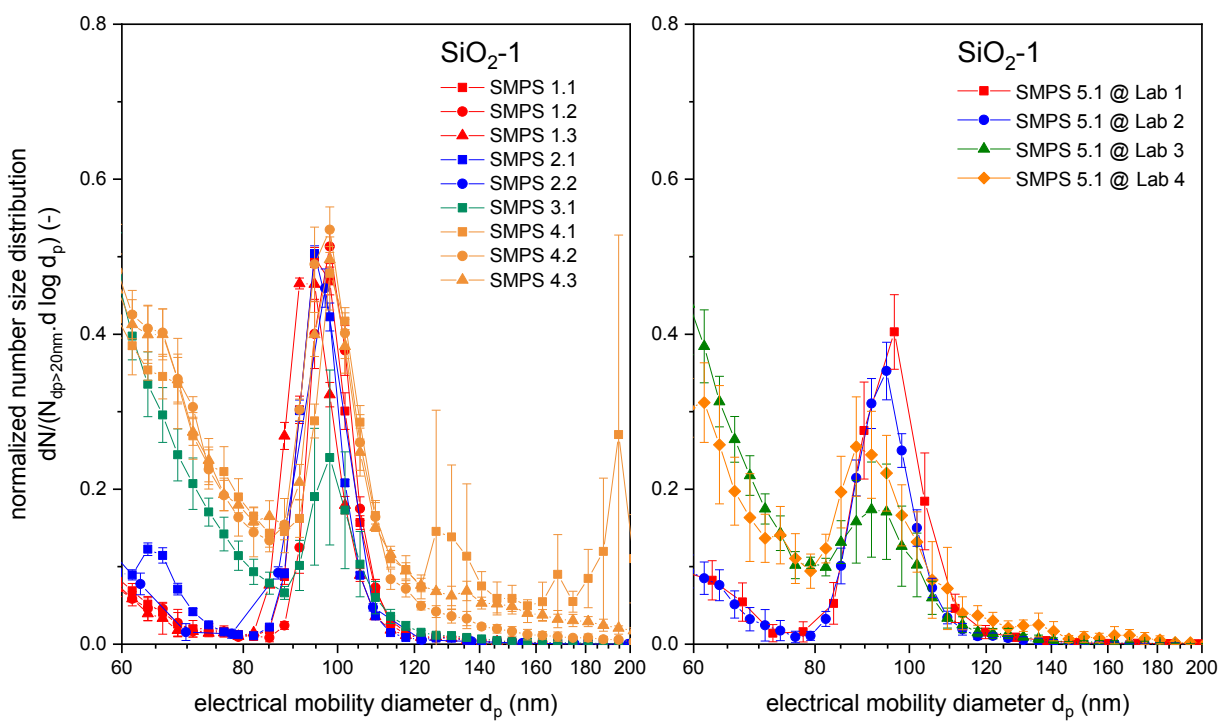

Fig3: Average particle number size distributions for the $\mathrm{SiO}_{2}-1$ sample. 

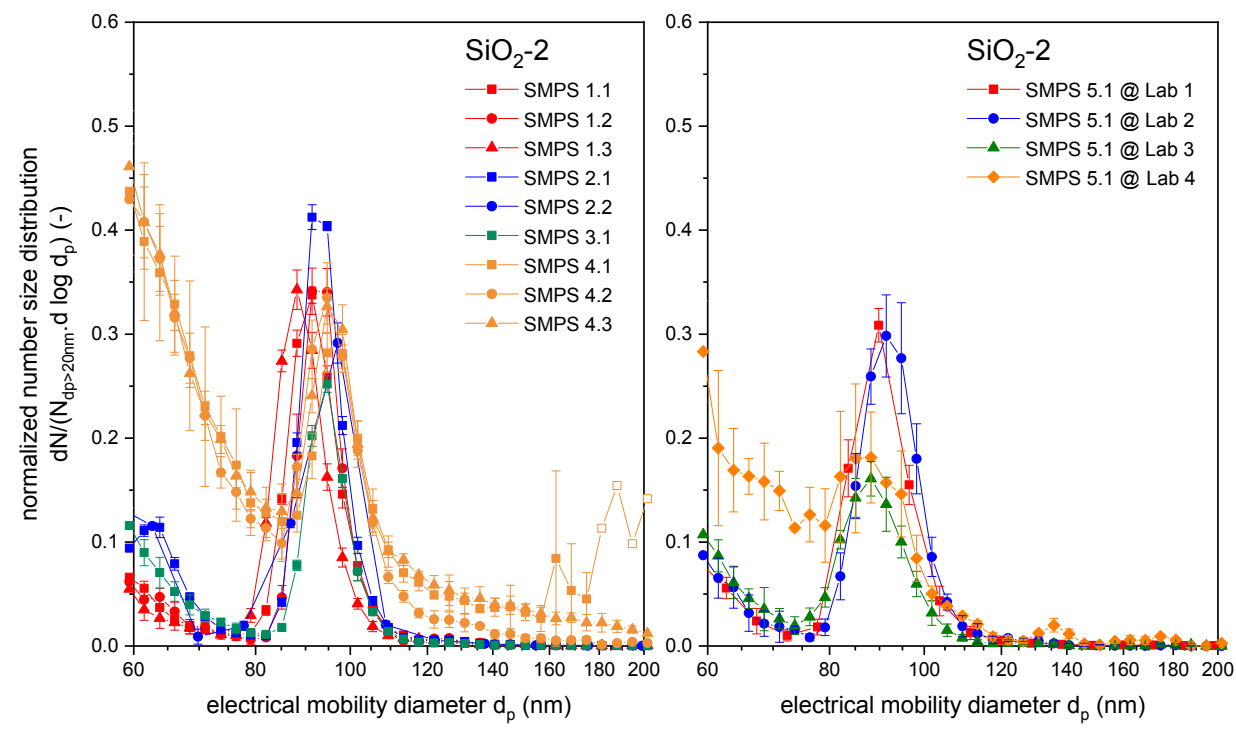

Fig4: Average particle number size distributions for the $\mathrm{SiO}_{2}-2$ sample.
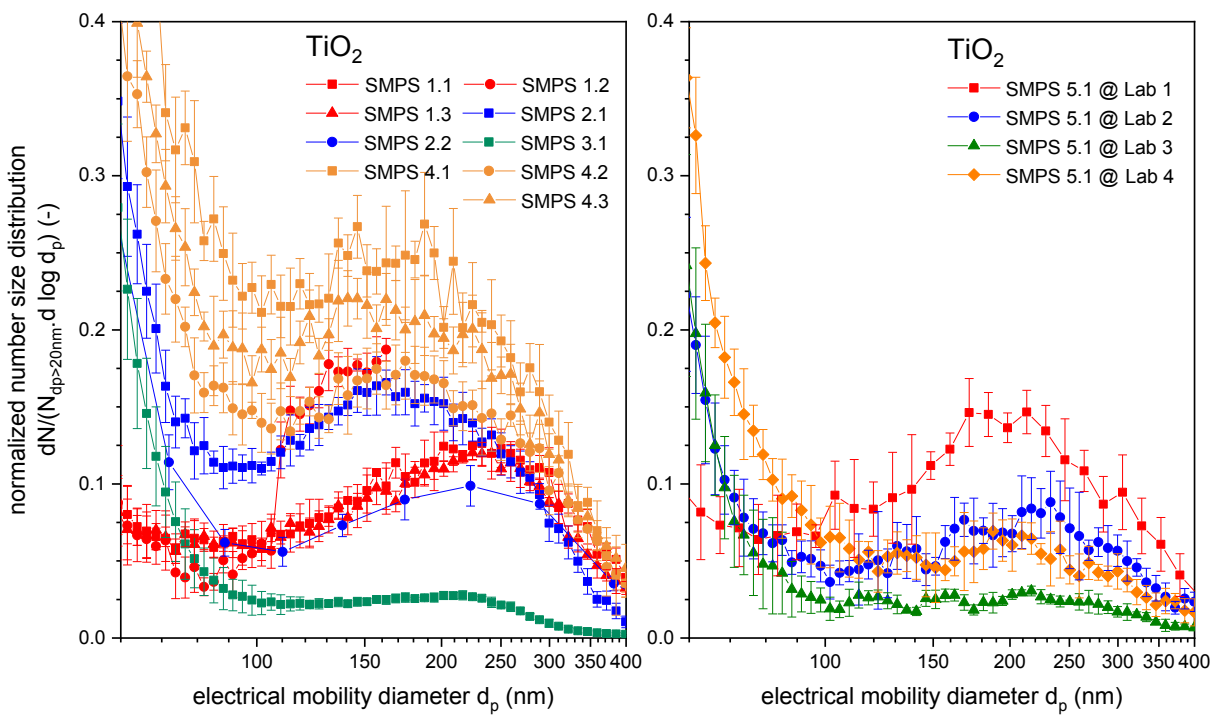

Fig5: Average particle number size distributions for the $\mathrm{TiO}_{2}$ sample.

5

From Fig5, greater result variability was obtained for $\mathrm{TiO}_{2}$. This was associated to the low $\mathrm{TiO}_{2}$ aerosol concentrations in number linked to the low $\mathrm{TiO}_{2}$ concentration in the provided stock sample. It is important to note that as sociated dilutions of stock sample were optimized deliberated by the consortium since the main objective of this study was to demonstrate the good laboratory practices using common resources in terms of aerosol generation and measurement set-ups and common sample preparation protocols. As an example, SMPS3.1 data in Fig2 shows a strong aerosol background due to water nebulisation. This is clearly presented in Fig8 (see appendix) in which a difference in terms of aerosol background due to the water-only nebulisation was observed between the morning and afternoon session during this study due to the cleaning of ultrapure water generation system. This highlights that laboratory practices are important to be considered for such study. 
Mean, modal and median diameters are commonly used to describe lognormal particle size distributions. Since overlapping size distributions are predominant in this study, it was chosen to take into account only modal diameters in data processing and thus to avoid any deconvolution of size distributions. It is worth mentioning that PNSD in Fig2-5 are presented as curves instead of rigorous histograms in order to improve their readability. Therefore, each point of the curves corresponds to the mid-point diameter of the associated size bin which is defined by the geometric mean diameter. Consequently, in a logarithmic scale, the modal diameter corresponds to the geometric mean diameter of the most frequent channel. Table 2 presents the average modal diameters measured by each laboratory and by the control SMPS (Lab 5) for the four samples involved in this study.

Table 2: Average (Av.) modal diameters measured by each SMPS for each laboratory and for the control SMPS (Lab 5) for each sample. Repeatability and reproducibility standard deviations (STDr (k=1) and $\operatorname{STD}_{\mathrm{R}}(\mathrm{k}=2)$ ) were calculated in accordance with the ISO 5725-2 (1994) standard.

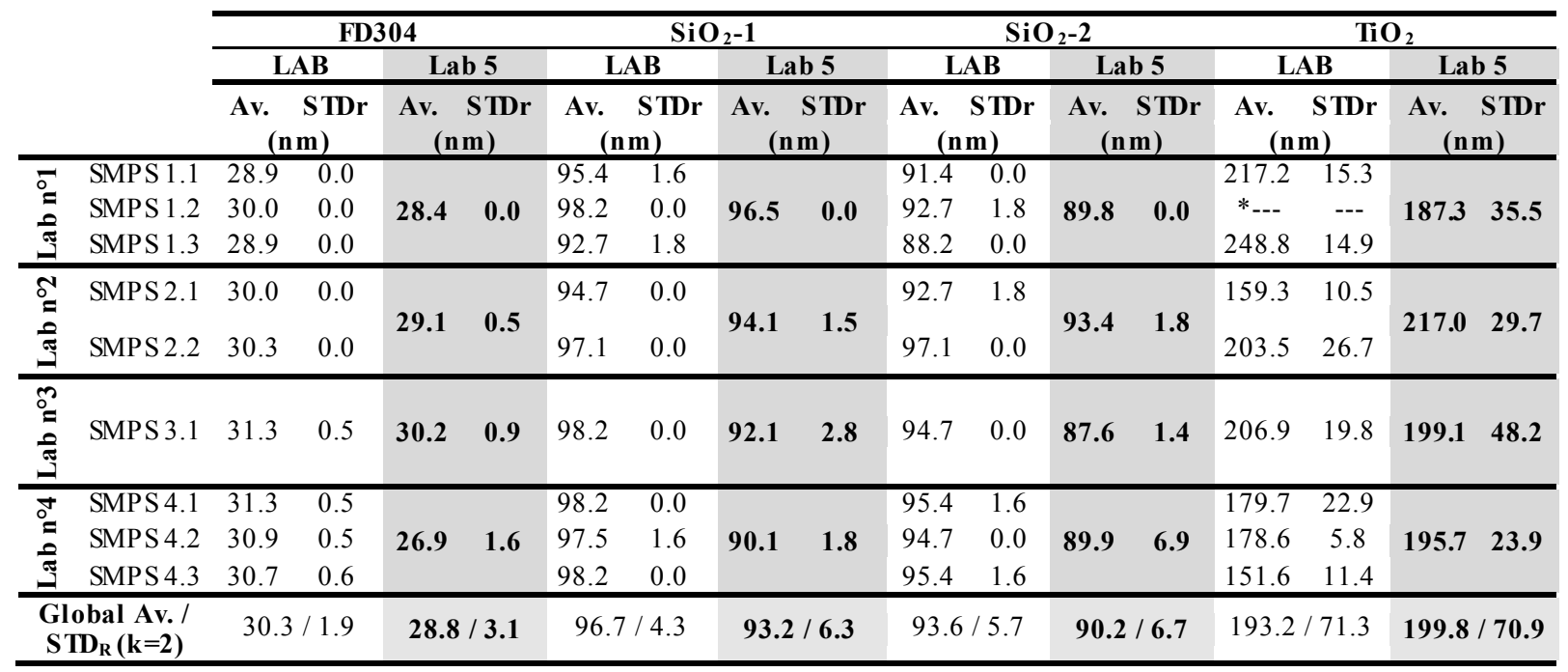

* not determined (due to inadapted size range [nano column])

Repeatability and reproducibility standard deviations (STDr and STD ${ }_{\mathrm{R}}$ ) were calculated in accordance with ISO 5725-2 (1994). For each sample, global average modal diameters (GAMD) were calculated by integrating all SMPS types from $\mathrm{Lab} \mathrm{n}^{\circ} 1$ to $\mathrm{Lab} \mathrm{n}^{\circ} 4$ and for the control SMPS (Lab $\mathrm{n}^{\circ} 5$ ) with reproducibility standard deviation $(k=2)$ reported as uncertainties. GAMD for [Lab1-4 / control SMPS] are calculated to be $[30.3 \pm 1.9 \mathrm{~nm} / 28.8 \pm 3.1 \mathrm{~nm}] ;[96.7 \pm 4.3 \mathrm{~nm} / 93.2 \pm 6.3 \mathrm{~nm}] ;[93.6 \pm 5.7 \mathrm{~nm} /$ $90.2 \pm 6.7 \mathrm{~nm}]$ and $[193.2 \pm 71.3 \mathrm{~nm} / 199.8 \pm 70.9 \mathrm{~nm}]$ for $\mathrm{FD} 304, \mathrm{SiO}_{2}-1, \mathrm{SiO}_{2}-2$ and $\mathrm{TiO}_{2}$ samples, respectively. SMPS measurements appear consistent for each laboratory especially for $\mathrm{SiO}_{2}$ samples which are characterized by relative $\mathrm{STD}_{\mathrm{R}}$ comprised between 4 and $11 \%$ while $\mathrm{TiO}_{2}$ samples are characterized by relative $\mathrm{STD}_{\mathrm{R}}$ around $35 \%$. By taking into account the control SMPS GAMD as reference values, a $\mathrm{Z}$ score performance test was performed for each SMPS and each sample as:

$$
Z=\frac{d_{\bmod _{S M P S i}}{ }^{-d_{\text {mod }}}{ }_{S M P S^{*}}}{S T D_{R}}
$$

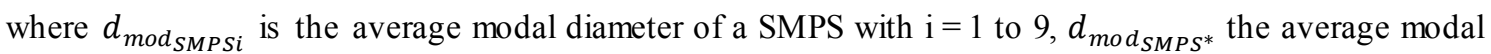
diameter of the control SMPS and $\mathrm{STD}_{\mathrm{R}}$ the estimated reproducibility standard deviation $(k=2)$.

SMPS performance was therefore assessed knowing that:

- $\quad$ absolute $Z$ score values $|Z|$ greater than 3 are considered to be unsatis factory values ("warning zone");

- $\quad 2<|Z| \leq 3$ are considered to be questionable values ("surveillance zone");

- $1<|z| \leq 2$ are coherent values and correspond to acceptable performance;

- $|Z| \leq 1$ are optimal values and correspond to excellent performance. 


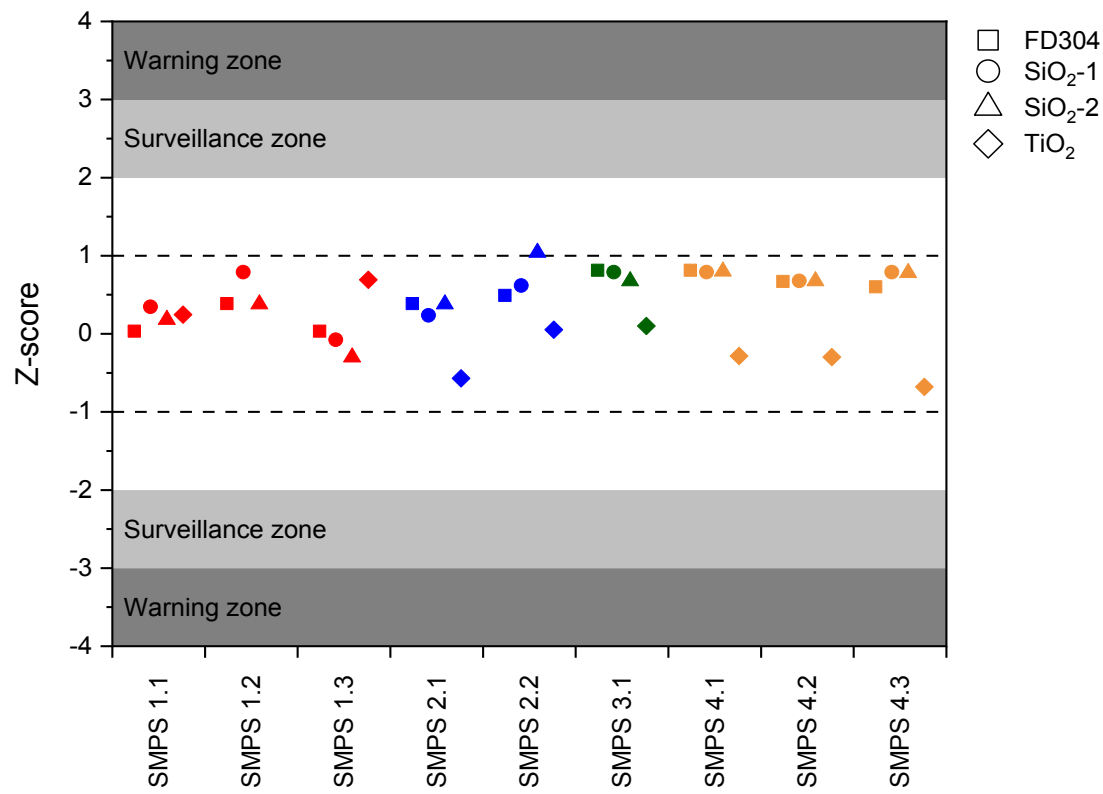

Fig6: Z Score calculation for the average modal diameters measured by each SMPS for the four samples (reference to the control SMPS)

Z score calculation for the average modal diameters measured by each SMPS for the four samples are presented in Fig6. All Z score values are comprised between -1 and 1 for this intercomparison exercise. This highlights the good practices and the ability of each laboratory to measure the modal diameters in connection with the use of provided protocols for each sample studied. In link with discussions in section 4.1, these results clearly show that modal diameter measurements especially for SMPS3.1 are not hindered despite the fact that, in this case, a high background associated to water nebulisation was observed.

\subsection{Comparison with electron microscopy measurements}

Considering scanning electron microscopy results associated with this intercomparison study, measured average modal diameters were found to be $26.0 \pm 0.3 \mathrm{~nm}, 93.3 \pm 1.2 \mathrm{~nm}, 93.6 \pm 1.2 \mathrm{~nm}$ and $95.0 \pm 0.7 \mathrm{~nm}$ for FD304, second modal diameter for $\mathrm{SiO}_{2}-1$ and $\mathrm{SiO}_{2}-2$ populations and $\mathrm{TiO}_{2}$ respectively. Such diameters measured by SEM correspond to projected area equivalent diameter. It should be noted that the ERM-FD304 certified reference material certificate indicates a modal diameter of $27.8 \pm 1.5 \mathrm{~nm}$ measured by electron microscopy (TEM and SEM) which is slightly higher (7\%) than the particle sizes measured by SEM in this study. However, both values are consistent by taking into account associated uncertainties.

According to the SMPS-based modal diameters gathered in Table 2 and by taking into account expanded uncertainty $(k=2)$, it can be concluded a satisfying agreement with SEM-based diameters, probably due to the spherical shape of $\mathrm{SiO}_{2}$ particles (Fig7A, B, C), except for the $\mathrm{TiO}_{2}$ sample. The default of accordance between SEM- and SMPS-based modal diameter measurements in the case of $\mathrm{TiO}_{2}$ sample can be due to the fact that primary particles were taken into account for SEM measurements (Fig7D), while SMPS measurements concern NOAAs due to the nebulization process of aqueous colloidal suspension. Indeed, Fig7E shows an example of $\mathrm{TiO}_{2}$ NOAAs sampled on a carbon TEM grid from an aerosol produced using the experimental setup presented in Fig 1 and using the Mini Particle Sampler (MPS) system (R'Mili et al, 2013). The latter clearly shows that airborne $\mathrm{TiO}_{2}$ particles have an agglomerated/aggregated morphology. 


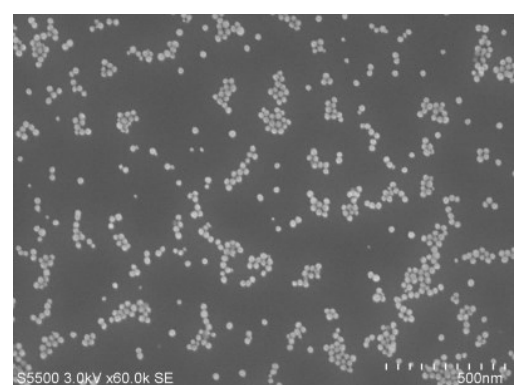

(A)

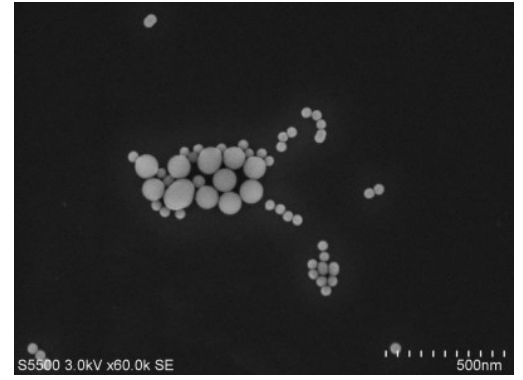

(C)

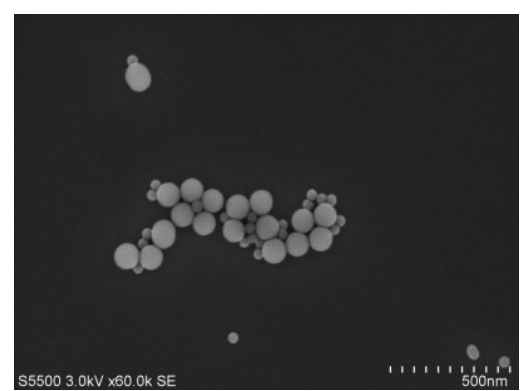

(B)

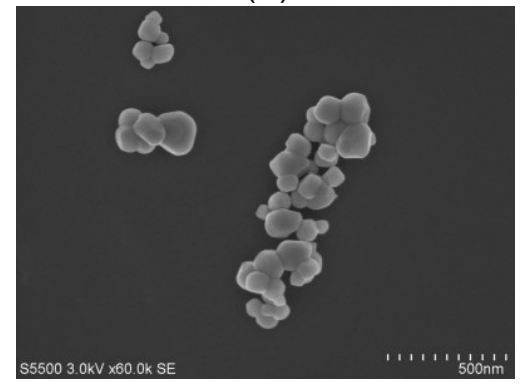

(D)

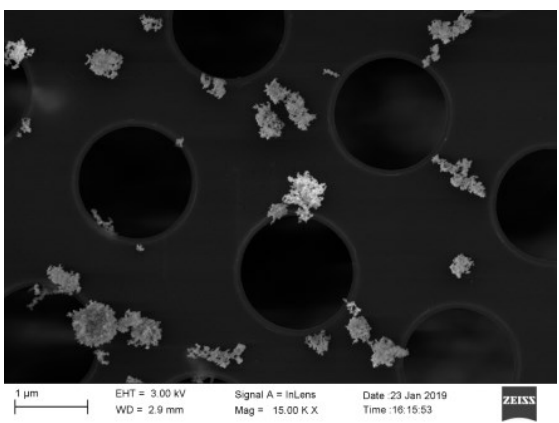

(E)

Fig7: SEM/TEM pictures of (A) - FD304, (B) - $\mathrm{SiO}_{2}-1,(\mathrm{C})-\mathrm{SiO}_{2}$-2, (D) - Food-grade $\mathrm{TiO}_{2}$ sample and (E) example of $\mathrm{TiO}_{2}$ aerosol sampled on a carbon TEM grid.

\section{3}

4

\section{Conclusions and outlooks}

This article presents the results from an intercomparison of good laboratory practices dedicated to nanoaerosols size measurements using harmonized experimental set-up and sample preparation protocols with an identified schedule and comparis on with SEM measurements. Global average modal diameters were calculated for each sample from all SMPS measurements, i.e. $30.3 \pm 1.9 \mathrm{~nm} ; 96.7 \pm 4.3 \mathrm{~nm}$; $93.6 \pm 5.7 \mathrm{~nm} ; 193.2 \pm 71.3 \mathrm{~nm}$ for sample ERM-FD304, $\mathrm{SiO}_{2}-1, \mathrm{SiO}_{2}-2$ and $\mathrm{TiO}_{2}$ respectively with reproducibility standard deviation $(\mathrm{k}=2)$ reported as expanded uncertainties and knowing that ERMFD304 sample is a certified reference material characterized by modal diameter of $27.8 \pm 1.5 \mathrm{~nm}$. Complementary scanning electron microscopy results were obtained and average modal diameters were found to be $26.0 \pm 0.3 \mathrm{~nm}, 93.3 \pm 1.2 \mathrm{~nm}, 93.6 \pm 1.2 \mathrm{~nm}$ and $95.0 \pm 0.7 \mathrm{~nm}$ for each sample respectively. Relative differences of $4 \%$ and $7 \%$ for SMPS and SEM measurements were obtained for the ERMFD304 modal diameter measurements. However, measurements are consistent by taking into account associated uncertainties. Such a study leads to the global validation of good laboratory practices in terms of aerosol generation and measurement set-ups involving SMPS since Z score calculations for the average modal diameters measured by each SMPS for the four samples were comprised between -1 and 1 . Such laboratory practices take into account the variation of technical parameters associated to the experimental set-up, environmental conditions and experimental operators.

As a perspective, new intercomparis on studies will be organized on the measurement of the aerosol size distribution by involving optical (WRAS, WELAS, Dusttrack), aerodynamic (APS, ELPI, cascade impactors) and electrical mobility sizers (SMPS, FMPS, DMS, nanoscan ...) by sending a same transportable reference aerosol generator to each participant in the same way of the work performed by Gaie-Levrel et al. (2017) about particle mass concentration. 
Asbach C, Kaminski H, Fissan H, Monz C, Dahmann D, Mülhopt S, Paur HR, Heinz JK, Herrmann F, Voetz M, Kuhlbusch T (2009) Comparis on of four mobility particle sizers with different time resolution for stationary exposure measurements, Journal of Nanoparticle Research 11:15931609.

Babick F, Mielke J, Wohlleben W, Weigel S, Hodoroaba V-D (2016a) How reliably can a material be classified as a nanomaterial? Available particle-sizing techniques at work, Journal of Nanoparticle Research 18(6).

Babick F, Mielke J, Hodoroaba V-D, Weigel S, Wohlleben W (2016b) Critical review manuscript with real world performance data for counting, ensemble and separating methods including in -build mathematical conversion to number distributions submitted for publication - NanoDefine Technical Report D3.3, in NanoDefine.

Bau S, Witschger O, Gensdarmes F, Thomas D (2013). Determining the count median diameter of nanoaerosols by simultaneously measuring their number and lung deposited surface area concentrations, J. Nanopart. Res. 15, 2104.

Bau S, Toussaint A, Payet R, Witschger O (2017) Performance study of various Condensation Particle Counters (CPCs): development of a methodology based on steady-state airborne DEHS particles and application to a series of handheld and stationary CPCs, Journal of Physics: Conference Series, IOP Publishing 838, pp.012002.

Bau S, Payet R, Tritscher T, Witschger O (2019). Intercomparison in the laboratory of various Condensation Particle Counters challenged by nanoaerosols in the range $6-460 \mathrm{~nm}$, Journal of Physics: Conference Series, 1323 012004. doi:10.1088/1742-6596/1323/1/012004

Club nanoMétrologie, (2019) http://www.club-nanométrologie.fr

Dahmann D, Riediger G, Schlatter J, Wiedensohler A, Carli S, Graff A, Grosser M, Hojgr M, Horn H-G, Jing L, Matter U, Monz C, Mosimann T, Stein H, Wehner B, Wieser U (2001) Intercomparison of Mobility Particle Sizers (MPS). Gefahrstoffe-Reinhaltung der Luft 61(10):423-428.

Ding YB, Stahlmecke B, Kaminski H, Jiang Y, Kuhlbusch TAJ, Riediker M (2016) Deagglomeration testing of airborne nanoparticle agglomerates: Stability analysis under varied aerodynamic shear and relative humidity conditions, Aerosol Science and Technology 50(11), pp 1253-1263.

Fissan H, Hummes D, Stratmann F, Büscher P, Neumann S, Pui DYH, Chen D (1996) Experimental comparis on of four differential mobility analyzers for nanometer aerosol measurements, Aerosol Science and Technology 24:1-13.

Fonseca AS, Viana M, Pérez N, Alastuey A, Querol X, Kaminski H, Todea AM, Monz C, Asbach C (2016) Intercomparison of a portable and two stationary mobility particle sizers for nanoscale aerosol measurements Aerosol Science and Technology 50:7,653-668, DOI: $10.1080 / 02786826.2016 .1174329$.

Franklin L, Bika A, Watts W, Kittelson D (2010) Comparison of Water and Butanol Based CPCs for Examining Diesel Combustion Aerosols, Aerosol Science and Technology, 44:8, 629-638, DOI: $10.1080 / 02786826.2010 .482112$.

Fuchs NA (1963) On the stationary charge distribution on aerosol particles in abipolar ionic atmosphere, Pure and Applied Geophysics, 56(1), 185-193.

Gaie-Levrel F, Bourrous S, Macé T (2017) Development of a Portable Reference Aerosol Generator (PRAG) for calibration of particle mass concentration measurements, Particuology, 37, DOI: 10.1016/j.partic.2017.06.005. 
Gómez-Moreno FJ, Alonso E, Artiñano B, Juncal-Bello V, Iglesias-Samitier S, Piñeiro Iglesias M, López Mahía P, Pérez N, Pey J, Ripoll A, Alastuey A, de la Morena BA, Rodríguez MIG, Sorribas M, Titos G, Lyamani H, Alados-Arboledas L, Latorre E, Tritscher T, Bischof OF (2015) Intercomparisons of Mobility Size Spectrometers and Condensation Particle Counters in the Frame of the Spanish Atmospheric Observational Aerosol Network, Aerosol Science and Technology, 49:777-785

Helsper C, Horn HG, Schneider F, Wehner B, Wiedensohler A (2008) Intercomparison of Five Mobility Size Spectrometers for Measuring Atmospheric Submicrometer Aerosol Particles. Gefahrstoffe Reinhaltung der Luft., 68:475-481

ICRP (1994) Publication 66: Human respiratory tract model for radiological protection, Oxford: Pergamon.

ISO 5725-2 (1994) Accuracy (trueness and precision) of measurement methods and results - Part 2: Basic method for the determination of repeatability and reproducibility of a standard measurement method.

Jeong C-H, Evans GJ (2009) Inter-comparison of a fast mobility particle sizer and a scanning mobility particle sizer incorporating an ultrafine water-based condensation particle counter, Aerosol Science and Technology 43:364-373

Joshi M, Sapra BK, Khan A, Tripathi SN, Shamjad PM, Gupta T, Mayya YS (2012) Harmonisation of nanoparticle concentration measurements using GRIMM and TSI scanning mobility particle sizers, Journal of Nanoparticle Research 14, 1268-1281.

Kallinger P, Steiner G, Szymanski WW (2012) Characterization of four different bipolar charging devices for nanoparticle charge conditioning, Journal of Nanoparticle Research, 14, 944-951.

Kaminski H, Kuhlbusch TAJ, Rath S, Götz U, Sprenger M, Wels D, Polloczek J, Bachmann V, Dziurowitz N, Kiesling HJ, Schwiegelsohn A, Monz C, Dahmann D, Asbach C (2013) Comparability of mobility particle sizers and diffusion chargers, Journal of Aerosol Science, 57, 156-178.

Khlystov A, Kos GPA, ten Brink HM, Mirme A, Tuch T, Roth C, Kreyling WG (2001) Comparability of Three Spectrometers for Monitoring Urban Aerosol, Atmospheric Environment 35:2045-2051.

Kulkarni P, Baron PA, Willeke K (Eds.) (2011) Aerosol measurement: principles, techniques, and applications, John Wiley \& Sons.

Lall AA, Friedlander SK (2006) On-line measurement of ultrafine aggregate surface area and volume distributions by electrical mobility analysis: I. Theoretical analysis. Journal of Aerosol Science 37:260-271.

Lawrence MG, Butler TM, Steinkamp J, Gurjar BR, Lelieveld J (2007) Regional pollution potentials of megacities and other major population centers, Atmos. Chem. Phys., 7, 3969-3987, doi:10.5194/acp-7-3969-2007.

Lee ES, Polidori A, Koch M, Fine PM, Mehadi A, Hammond D, Wright JN, Miguel AH, Ayala A, Zhu Y (2013) Water-based condensation particle counters comparison near a major freeway with significant heavy-duty diesel traffic, Atmospheric Environment 68 151e161

Leskinen J, Joutsensaari J, Lyyränen J, Koivisto J, Ruusunen J, Järvelä M, Jokiniemi J (2012) Comparison of nanoparticle measurement instruments for occupational health applications, Journal of Nanoparticle Research, 14(2), 718.

Motzkus C, Macé T, Gaie-Levrel F, Ducourtieux S, Delvallée A, Dirscherl K et al. (2013) Size characterization of airborne $\mathrm{SiO}_{2}$ nanoparticles with on-line and off-line measurement techniques: an interlaboratory comparison study, Journal of Nanoparticle Research 15(10). 
Nicosia A, Manodori L, Trentini A, Ricciardelli I, Bacco D, Poluzzi V, Di Matteo L, Belosi F (2018) Field study of a soft X-ray aerosol neutralizer combined with electrostatic classifiers for nanoparticle size distribution measurements, Particuology 37 99-106.

Nicosia A, Belosi F, Vazquez B (2014). Application of the soft X-Ray TSI advanced aerosol neutralizer to aerosol measurements made by a Grimm SMPS, Atmospheric and Climate Sciences 4(4), 636-641.

R'Mili B, Le Bihan O, Dutouquet C, Aguerre-Chariol O, Frejafon E (2013) Particle Sampling by TEM Grid Filtration, Aerosol Science and Technology, Taylor \& Francis 47 (7), pp.767-775.

Rodrigue J, Dhaniyala S, Ranjan M, Hopke PK (2007) Performance comparis on of scanning electrical mobility spectrometers, Aerosol Science and Technology 41:360-368.

Stolzenburg MR, McMurry PH (2008). Equations governing single and tan-dem DMA configurations and a new lognormal approximation to the transfer function, Aerosol Science and Technology, 42(6), $421-432$.

Tigges L, Wiedensohler A, Weinhold K, Gandhi J, Schmid H-J (2015) Bipolar Charge Distribution of a Soft X-Ray Diffusion Charger, Journal of Aerosol Science 90:77-86.

Vedal S, Dutton SJ (2006). Wildfire air pollution and daily mortality in a large urban area, Environ. Res. $102,29-35$.

Watson JG, Chow JC, Sodeman DA, Lowenthal DH, Chang MCO, Park K, Wang X (2011) Comparis on of four scanning mobility particle sizers at the Fresno Supersite. Particuology, 9, 204-209.

Wiedensohler A, Wiesner A, Weinhold K, Birmili W, Hermann M, Merkel M, Müller T, Pfeifer S, Schmidt A, Tuch T, Velarde F, Quincey P, Seeger S, Nowak A, (2017) Mobility particle size spectrometers: Calibration procedures and measurement uncertainties, Aerosol Science and Technology, 52: 146-164.

Wiedensohler A, Birmili W, Nowak A et al. (2012) Mobility particle size spectrometers: harmonization of technical standards and data structure to facilitate high quality long-term observations of atmospheric particle number size distributions, Atmos Meas Technol 5:657-685.

Wiedensohler A, Aalto P, Covert D, Heintzenberg J, McMurry P (1993) Intercomparison of three methods to determine size distributions of ultrafine aerosols with low number concentrations, $\mathrm{J}$ Aerosol Sci 24:551-554.

Wiedensohler A, Lütkemeier E, Feldpausch M, Helsper C (1986), Investigation of the Bipolar Charge distribution at various gas conditions, Journal of Aerosol Science, 17:413.

Wiedensohler A (1988) Technical Note: An approximation of the Bipolar Charge distribution for particles in the submicron range, Journal of Aerosol Science, 19:3/387-389. 
1 Appendix:
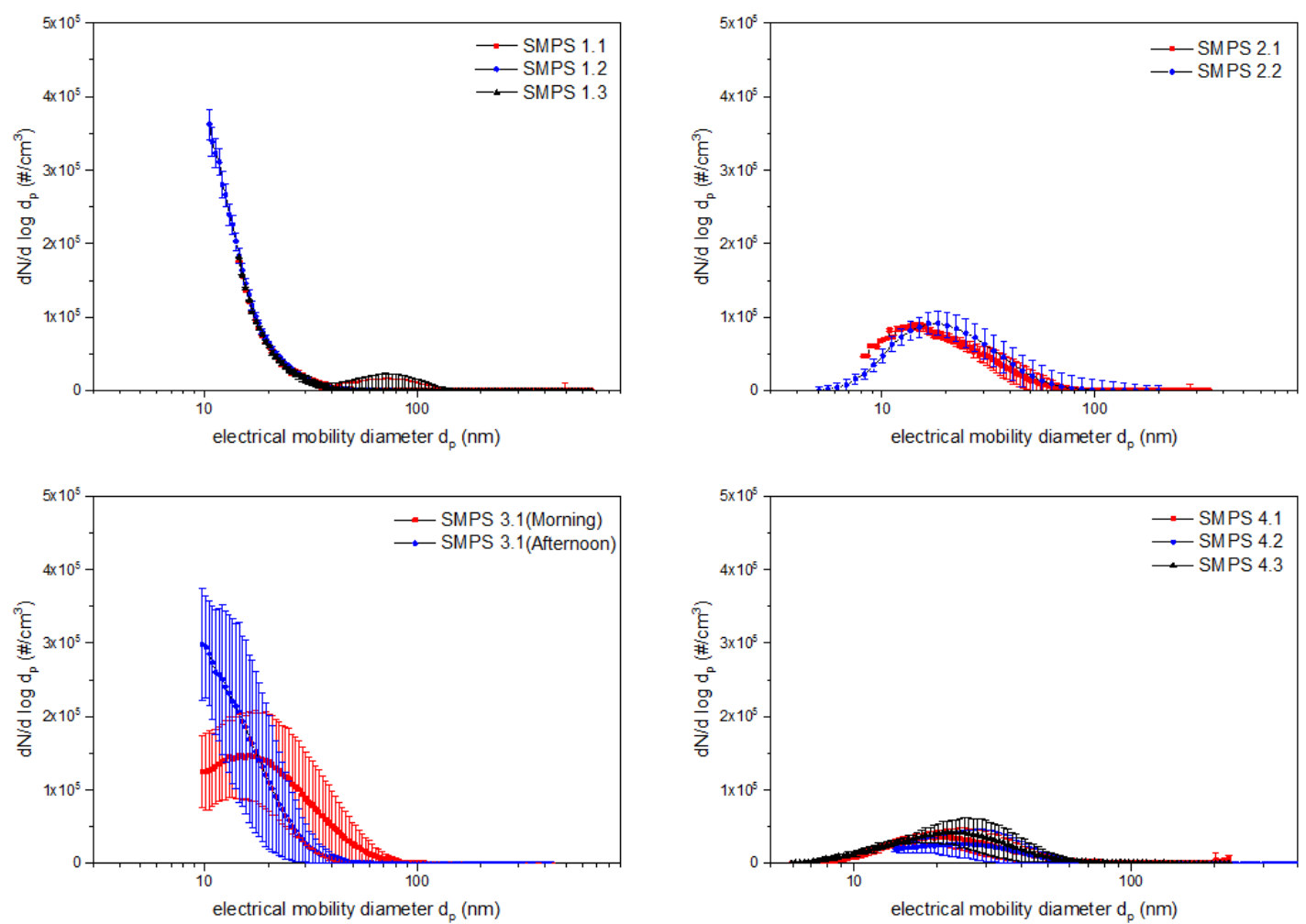

3 Fig8: Average PNSD for aerosols generated from the water nebulization only, by each participant and 4 measured by each involved SMPS. 